\title{
Functional outcomes from a head-to-head, randomized, double-blind trial of lisdexamfetamine dimesylate and atomoxetine in children and adolescents with attention-deficit/hyperactivity disorder and an inadequate response to methylphenidate
}

\author{
Peter Nagy ${ }^{1} \cdot$ Alexander Häge $^{2} \cdot$ David R. Coghill $^{3} \cdot$ Beatriz Caballero $^{4} \cdot$ Ben Adeyi $^{5}$. \\ Colleen S. Anderson ${ }^{5}$ Vanja Sikirica ${ }^{5} \cdot$ Esther Cardo $^{6}$
}

Received: 2 September 2014 / Accepted: 2 April 2015 / Published online: 22 May 2015

(c) The Author(s) 2015. This article is published with open access at Springerlink.com

\begin{abstract}
Attention-deficit/hyperactivity disorder (ADHD) is associated with functional impairments in multiple domains of patients' lives. A secondary objective of this randomized, active-controlled, head-to-head, double-blind, dose-optimized clinical trial was to compare the effects of lisdexamfetamine dimesylate (LDX) and atomoxetine (ATX) on functional impairment in children and adolescents with ADHD. Patients aged 6-17 years with an ADHD Rating Scale IV total score $\geq 28$ and an inadequate response to methylphenidate treatment (judged by investigators) were randomized (1:1) to once-daily LDX or ATX for 9 weeks.
\end{abstract}

ClinicalTrials.gov identifier: NCT01106430. Study funded by Shire Development LLC.

P. Nagy and A. Häge contributed equally to this work.

Electronic supplementary material The online version of this article (doi:10.1007/s00787-015-0718-0) contains supplementary material, which is available to authorized users.

Esther Cardo

ecardojalon@gmail.com

1 Vadaskert Child and Adolescent Psychiatry Hospital and Outpatient Clinic, Budapest, Hungary

2 Paediatric Psychopharmacology, Department of Child and Adolescent Psychiatry and Psychotherapy, Central Institute of Mental Health, Medical Faculty Mannheim, University of Heidelberg, Mannheim, Germany

3 Division of Neuroscience, University of Dundee, Dundee, UK

4 Shire, Eysins, Switzerland

5 Shire, Wayne, PA, USA

6 Neuropaediatric Unit, Son Llàtzer Hospital and Research Institute on Health Sciences, University of the Balearic Islands, Palma De Mallorca, Spain
Parents/guardians completed the Weiss Functional Impairment Rating Scale-Parent Report (WFIRS-P) at baseline and at week 9 or early termination. $p$ values were nominal and not corrected for multiple comparisons. Of 267 randomized patients, 200 completed the study (LDX 99, ATX 101). At baseline, mean WFIRS-P total score in the LDX group was 0.95 [standard deviation (SD) $0.474 ; 95 \%$ confidence interval (CI) $0.87,1.03$ ] and in the ATX group was $0.91(0.513 ; 0.82,1.00)$. Scores in all WFIRS-P domains improved from baseline to endpoint in both groups, with least-squares mean changes in total score of $-0.35(95 \%$ CI $-0.42,-0.29)$ for LDX and $-0.27(-0.33,-0.20)$ for ATX. The difference between LDX and ATX was statistically significant $(p<0.05)$ for the Learning and School (effect size of LDX vs ATX, 0.43) and Social Activities (0.34) domains and for total score (0.27). Both treatments reduced functional impairment in children and adolescents with ADHD; LDX was statistically significantly more effective than ATX in two of six domains and in total score.

Keywords Atomoxetine - Attention-deficit/hyperactivity disorder · Functional impairment · Lisdexamfetamine dimesylate - Weiss Functional Impairment Rating Scale-Parent Report

\section{Introduction}

Attention-deficit/hyperactivity disorder (ADHD) is characterized by the core symptoms of inattention, hyperactivity, and impulsivity. ADHD is also associated with substantial functional impairments that can affect social, academic, and occupational activities throughout life [6]. Indeed, the Diagnostic and Statistical Manual of Mental Disorders, 4th Edition, Text Revision (DSM-IV-TR) [3], the recent 
5th edition of the DSM (DSM-5) [2], and the International Classification of Diseases, 10th revision [43] all specify evidence of functional impairment as a diagnostic criterion for ADHD. The impact of such impairment on a patient's daily life commonly provides the motivation to seek medical treatment [37]. ADHD treatment should, therefore, aim not only to improve symptoms but also to reduce functional impairment. This is reflected in European regulatory guidance for clinical trials of ADHD medications, which state that such trials should include a functional outcome measure [30].

Lisdexamfetamine dimesylate (LDX) is a long-acting prodrug psychostimulant. Clinical trials in children, adolescents, and adults have shown LDX to be effective in the treatment of ADHD and to have a tolerability profile consistent with that of psychostimulant therapy [7, 8, 22, 31]. LDX is approved as a first-line treatment for ADHD in the USA, Canada, Brazil, and Australia and is the first long-acting amfetamine-based medication to be approved in Europe, where it is licensed in select countries for the treatment of children and adolescents with ADHD when response to previous methylphenidate (MPH) treatment is considered clinically inadequate by the supervising specialist.

Study SPD489-317 (ClinicalTrials.gov NCT01106430) was a 9-week, head-to-head, randomized, double-blind trial comparing LDX with atomoxetine (ATX), a non-stimulant noradrenaline reuptake inhibitor [27]. The study was conducted in a population of children and adolescents with ADHD in Europe and North America who were judged to have responded inadequately to MPH therapy (defined in 'Methods'). The primary efficacy outcome was the time to clinical response [defined as a Clinical Global ImpressionsImprovement (CGI-I) score of 1 (very much improved) or 2 (much improved)]. This was significantly shorter for patients receiving LDX than for those receiving ATX [12.0 days (95\% confidence interval (CI) 8.0, 16.0) vs 21.0 days $(15.0,23.0) ; p=0.001]$ [27].

Secondary efficacy outcomes revealed that significantly higher proportions of patients in the LDX group exhibited a clinical response (CGI-I score of 1 or 2) compared with those in the ATX group and that patients receiving LDX showed significantly greater improvements in ADHD symptoms (assessed using the ADHD Rating Scale IV [ADHDRS-IV]) than those receiving ATX, at every study visit in weeks 1-9 [27]. Assessment of these outcomes used the 'last observation carried forward' approach to missing data. The safety and tolerability profiles of both treatments were consistent with findings from previous clinical trials [27].

Here, we report the effects of LDX and ATX treatment on functioning in study SPD489-317, as measured using a disorder-specific instrument, the Weiss Functional Impairment Rating Scale-Parent Report (WFIRS-P) [29].
This was a pre-specified secondary objective of study SPD489-317.

\section{Methods}

This double-blind, randomized, active-controlled, parallelgroup clinical trial was conducted between June 2010 and July 2012 at 51 sites in Europe, the USA, and Canada. The protocol was approved by an institutional review board, an independent ethics committee, or a regulatory agency at each centre, and the study was conducted in accordance with the International Conference on Harmonisation of Good Clinical Practice guideline and with local ethical and legal requirements. Full details of the study design have been published previously [27].

\section{Study population}

Patients aged 6-17 years were eligible for enrolment if they met the DSM-IV-TR criteria for a primary diagnosis of ADHD, had a baseline ADHD-RS-IV total score of 28 or higher (indicating at least moderately severe symptoms), and had previously experienced (or were experiencing) an inadequate response to MPH treatment. 'Inadequate response' was judged by investigators and included, but was not limited to: variable or incomplete symptom control; inadequate duration of action; and the potential (in the investigator's opinion) for the patient to benefit clinically from an alternative to MPH treatment. Patients were excluded if they had been exposed to amfetamine or ATX previously, if they had experienced intolerable side effects with previous MPH treatment, or if their symptoms were well controlled with acceptable tolerability on their current ADHD medication. Patients were also excluded if they had failed to respond to more than one previous course of MPH (defined as worsened, unchanged, or minimally improved symptoms) or if they had previously been treated with more than one formulation of MPH [except short-term ( $\leq 4$ weeks) dose titration with immediate-release MPH, provided they experienced an adequate response]. Furthermore, patients were excluded if they had a comorbid psychiatric diagnosis with significant symptoms or other symptomatic manifestations (e.g. agitated states, marked anxiety, or tension) that contraindicated treatment with LDX or ATX in the opinion of the investigator. Patients with a conduct disorder were also excluded, but oppositional defiant disorder was not exclusionary.

\section{Study design}

After discontinuing any previous psychoactive medication for a 7-day washout period, patients were randomized 
in a 1:1 ratio to receive once-daily treatment with LDX or ATX for 9 weeks. Doses of LDX and ATX were adjusted at weekly intervals over a 4-week period until an 'acceptable' response was achieved; this was defined as a reduction of at least $30 \%$ in ADHD-RS-IV total score from baseline and a CGI-I score of 1 or 2 (very much improved or much improved) with tolerable side effects [27, 28, 33]. Patients in the LDX group initially received $30 \mathrm{mg} /$ day, and if required, the dose was titrated to $50 \mathrm{mg} /$ day and then to $70 \mathrm{mg} /$ day. Patients in the ATX group weighing $70 \mathrm{~kg}$ or more initially received $40 \mathrm{mg} / \mathrm{day}$, and if required, the dose was titrated to $80 \mathrm{mg} /$ day and then to $100 \mathrm{mg} /$ day. Patients in the ATX group weighing less than $70 \mathrm{~kg}$ initially received approximately $0.5 \mathrm{mg} / \mathrm{kg} / \mathrm{day}$, and if required, the dose was titrated to a final target dose of $1.2 \mathrm{mg} / \mathrm{kg} /$ day, with a maximum permitted dose of $1.4 \mathrm{mg} / \mathrm{kg} /$ day.

\section{Functional impairment assessments}

Change in functional impairment, assessed using the WFIRS-P, was a secondary efficacy outcome of the study (the primary efficacy and safety outcomes have been published previously [27]). The WFIRS-P was completed by each patient's parents or legal guardians at baseline (week 0) and either at week 9 or at an early termination visit attended by patients who withdrew from the study.

The WFIRS-P was designed to provide a disorder-specific measure of functioning in children and adolescents with ADHD and has been shown to have good internal consistency and moderate convergent validity with other instruments [29]. The questionnaire comprises 50 items, grouped into six domains (Family, Learning and School, Life Skills, Child's Self-Concept, Social Activities, and Risky Activities). Each item relates to the previous month and is scored on a four-point Likert scale $(0=$ never or not at all; $1=$ sometimes or somewhat; $2=$ often or much; $3=$ very often or very much) or recorded as not applicable [29]. This study used an early version of the WFIRS-P [41]; the current version refers to the Learning and School domain as the School domain [40]. Higher WFIRS-P scores indicate more severe functional impairment.

Data have not yet been published on the minimum changes in WFIRS-P scores from baseline to endpoint within a treatment group that correspond to clinically relevant improvements in functional impairment in patients with ADHD. One-half the standard deviation (SD) at baseline $[34,44]$ has been recommended as a value for the minimum clinically important difference in WFIRS$\mathrm{P}$ scores by the developers of the instrument and has been used in previous studies [32]. To our knowledge, no data exist on minimum clinically important differences between two active medications in improvements in WFIRS-P scores.

\section{Statistical analysis}

Sample size was calculated as previously described and was based on the primary efficacy outcome of time to clinical response (and not on any secondary outcome) [27]. WFIRS-P data were analysed for the Full Analysis Set, which comprised all patients who were randomized and received at least one dose of study medication, and which was based on the intention-to-treat principle. Baseline and week 9 scores were the observed values; endpoint was defined as the last on-treatment, post-baseline visit with a valid WFIRS-P assessment (endpoint is therefore equivalent to 'last observation carried forward' at week 9).

Changes in WFIRS-P total or domain scores from baseline to week 9 and endpoint were analysed using an analysis of covariance (ANCOVA) model with treatment group and country as fixed effects and baseline score as a covariate. The model did not include interaction terms. A formal statistical comparison between LDX and ATX for the leastsquares (LS) mean change in WFIRS-P total and domain scores from baseline to endpoint was pre-specified in the study protocol. A formal statistical analysis of the change from baseline to week 9 or endpoint within each treatment group was not pre-specified, but was performed ad hoc, and was not therefore protected against type I error. No adjustment of $p$ values for multiple comparisons was pre-specified or performed for secondary efficacy variables; $p$ values less than 0.05 should therefore be regarded as representing nominal statistical significance. Effect sizes were calculated as the difference in LS mean change in scores from baseline between the LDX and ATX groups divided by the root mean square error obtained from the ANCOVA model. Effect sizes of 0.2, 0.5, and 0.8 are considered to indicate small, medium, and large differences between treatment groups, respectively, and are more often used for comparisons of an active drug versus placebo than of one active drug versus another [25].

\section{Results}

\section{Patient disposition and baseline characteristics}

Patients $(n=267)$ were enrolled at 51 study sites in the USA $(n=138)$, Germany $(n=42)$, Canada $(n=35)$, Spain $(n=22)$ Hungary $(n=20)$, Sweden $(n=6)$, Belgium $(n=2)$, Italy $(n=1)$, and Poland $(n=1)$. Of 267 patients randomized (LDX, $n=133$; ATX, $n=134$ ), 262 received at least one dose of study drug and were included in the Full Analysis Set (LDX, $n=127$; ATX, $n=135$ ). Of these, 200 (74.9\%) completed the study (LDX, $n=99$; ATX, $n=101)$. Based on the intention-to-treat principle, one patient who was randomized to ATX but received LDX 
Table 1 WFIRS-P total and domain scores at baseline, week 9, and endpoint, mean (SD)

\begin{tabular}{|c|c|c|c|c|c|c|}
\hline & \multicolumn{2}{|l|}{ Baseline } & \multicolumn{2}{|l|}{ Week 9} & \multicolumn{2}{|l|}{ Endpoint } \\
\hline & $\begin{array}{l}\text { LDX } \\
(124 \leq n \leq 127)\end{array}$ & $\begin{array}{l}\text { ATX } \\
(128 \leq n \leq 135)\end{array}$ & $\operatorname{LDX}(92 \leq n \leq 95)$ & $\operatorname{ATX}(93 \leq n \leq 97)$ & $\begin{array}{l}\text { LDX } \\
(102 \leq n \leq 107)\end{array}$ & $\begin{array}{l}\text { ATX } \\
(110 \leq n \leq 114)\end{array}$ \\
\hline Family & $1.18(0.733)$ & $1.11(0.817)$ & $0.66(0.562)$ & $0.75(0.635)$ & $0.71(0.607)$ & $0.80(0.684)$ \\
\hline Learning and school & $1.20(0.657)$ & $1.19(0.671)$ & $0.54(0.444)$ & $0.69(0.551)$ & $0.56(0.450)$ & $0.72(0.553)$ \\
\hline Life skills & $1.07(0.496)$ & $1.02(0.571)$ & $0.70(0.447)$ & $0.66(0.450)$ & $0.73(0.472)$ & $0.69(0.483)$ \\
\hline Child's self-concept & $0.83(0.807)$ & $0.72(0.830)$ & $0.41(0.545)$ & $0.39(0.520)$ & $0.45(0.583)$ & $0.44(0.652)$ \\
\hline Social activities & $0.82(0.663)$ & $0.83(0.702)$ & $0.43(0.427)$ & $0.61(0.530)$ & $0.46(0.468)$ & $0.61(0.544)$ \\
\hline Risky activities & $0.44(0.434)$ & $0.39(0.376)$ & $0.22(0.228)$ & $0.26(0.257)$ & $0.23(0.244)$ & $0.27(0.260)$ \\
\hline Total & $0.95(0.474)$ & $0.91(0.513)$ & $0.51(0.308)$ & $0.59(0.401)$ & $0.54(0.333)$ & $0.62(0.412)$ \\
\hline
\end{tabular}

Data are presented as mean (standard deviation). Higher scores indicate greater impairment. Baseline and week 9 scores are based on observed values. Endpoint was defined as the last on-treatment, post-baseline visit with a valid assessment. Numbers of observations $(n)$ were in the ranges indicated, depending on domain. These data were not analysed statistically

ATX atomoxetine, LDX lisdexamfetamine dimesylate, $n$ number of observations, WFIRS-P Weiss Functional Impairment Rating Scale-Parent Report

in error was included in the ATX group in the Full Analysis Set. The most common reasons for discontinuation in the LDX group were adverse events $(n=8 ; 6.0 \%)$ and withdrawal by the patient $(n=8 ; 6.0 \%)$; the most common reasons in the ATX group were lack of efficacy $(n=13$; $9.7 \%)$ and adverse events $(n=10 ; 7.5 \%)$.

Baseline demographic characteristics were similar in both treatment groups [27]. The mean age of patients at baseline was 10.6 years (SD 2.93); $74.0 \%$ of patients were children (aged 6-12 years), and $75.2 \%$ were male. In both treatment groups, the most frequently reported reason for an inadequate response to previous MPH treatment was lack of efficacy [LDX 96/127 (75.6\%), ATX 106/135 $(78.5 \%)$ ]. The mean optimal dose during the dose-maintenance phase (the dose that was dispensed from visit 4) was $52.5 \mathrm{mg} /$ day $(\mathrm{SD}, 16.10$ ) in the LDX group [28/128 (21.9\%), 36/128 (28.1\%), and 41/128 (32.0\%) patients received 30, 50, and $70 \mathrm{mg} /$ day, respectively] and $40.2 \mathrm{mg} /$ day (20.05) in the ATX group [of patients weighing $<70$ $\mathrm{kg}, 15 / 134(11.2 \%)$ and 95/134 (70.9\%) received 0.5 and $1.2 \mathrm{mg} / \mathrm{kg} /$ day, respectively; of patients weighing $\geq 70 \mathrm{~kg}$, 2/134 (1.5\%), 1/134 (0.7\%), and 4/134 (3.0\%) patients received 40,80 , and $100 \mathrm{mg} /$ day, respectively] [27].

\section{WFIRS-P scores at baseline}

At baseline, mean WFIRS-P total scores and scores in each individual domain were similar across both treatment groups (Table 1; Figure S1). Mean WFIRS-P total scores were 0.95 (SD $0.474 ; 95 \%$ CI $0.87,1.03$ ) in the LDX group and 0.91 (SD 0.513; $95 \%$ CI 0.82, 1.00) in the ATX group (Table 1). Baseline data were not tested statistically for equivalence between treatment groups, but, in all domains and in total score, the mean score in the LDX group lay within the $95 \%$ CI of the mean in the ATX group and vice versa (Figure S1).

In both groups, the highest mean scores (indicating the greatest degree of impairment) were seen in the Family domain [LDX 1.18 (SD 0.733), ATX 1.11 (0.817)] and the Learning and School domain [LDX 1.20 (SD 0.657), ATX 1.19 (0.671)] (Table 1).

\section{Difference between LDX and ATX groups in change in WFIRS-P scores from baseline to endpoint}

Compared with ATX treatment, LDX treatment was associated with numerically greater LS mean decreases from baseline to endpoint in WFIRS-P total score and scores in all domains except Life Skills. These differences between the LDX and ATX groups were statistically significant for total score $[p=0.046$; effect size 0.27 (LDX vs ATX)] and the domains of Learning and School $[p=0.002$; effect size 0.43 (LDX vs ATX)] and Social Activities $[p=0.014$; effect size 0.34 (LDX vs ATX)] (Fig. 1).

\section{Change in WFIRS-P scores from baseline to week 9 and endpoint within treatment group}

Mean WFIRS-P total and domain scores at week 9 and endpoint are shown in Table 1. Both treatments were associated with statistically significant reductions from baseline (indicating improvement) in LS mean WFIRS-P total score at week 9 [LDX -0.37 (95\% CI $-0.44,-0.30)$, ATX $-0.30(-0.36,-0.23)$; both $p<0.001]$ and at endpoint [LDX -0.35 (95\% CI $-0.42,-0.29)$, ATX $-0.27(-0.33$, -0.200 ); both $p<0.001$ ] (Fig. 2). Reductions from baseline in LS mean scores in all WFIRS-P domains were also significant at week 9 and endpoint in both treatment groups 


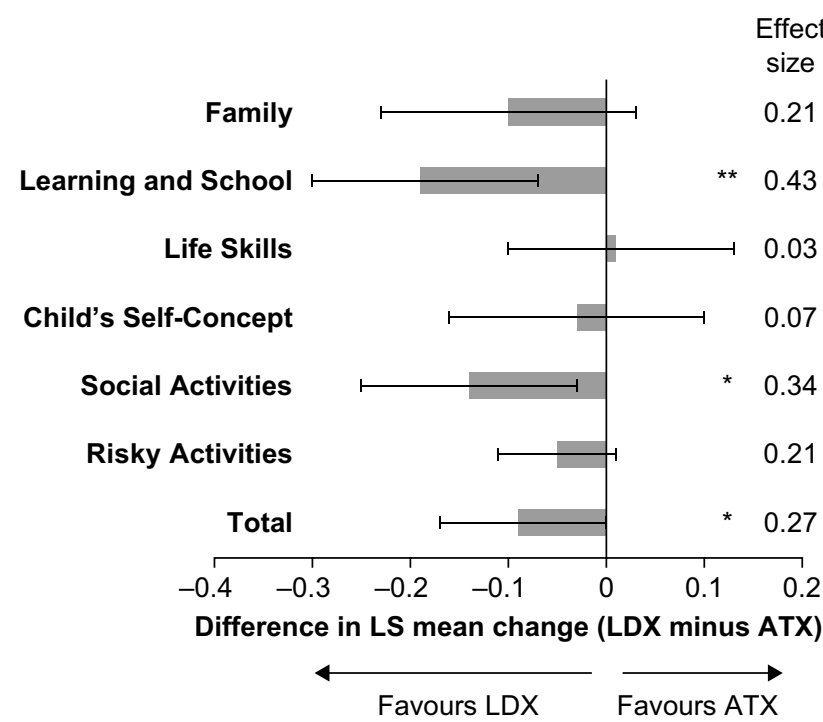

Fig. 1 Difference between LDX and ATX groups in change in WFIRS-P scores from baseline to endpoint. Histogram shows the difference between treatment groups in LS mean change from baseline to endpoint. Error bars show $95 \%$ confidence intervals. Endpoint was defined as the last on-treatment, post-baseline visit with a valid assessment. Effect size is the difference in LS mean change divided by root mean square error. $p$ values are nominal and were not adjusted for multiple comparisons. ${ }^{*} p<0.05$ and ${ }^{*} p<0.01 \mathrm{LDX}$ versus ATX (pre-specified analysis). ATX atomoxetine, $L D X$ lisdexamfetamine dimesylate, $L S$ least-squares, WFIRS-P Weiss Functional Impairment Rating Scale-Parent Report
( $p<0.001$; Fig. 2). The greatest reductions were seen in the Learning and School domain at week 9 [LDX -0.63 (95\% CI -0.73, -0.54), ATX -0.47 (-0.57, -0.38)] and at endpoint [LDX $-0.62(95 \%$ CI $-0.71,-0.52)$, ATX $-0.43(-0.52,-0.34)]$.

In both the LDX and ATX treatment groups, the reductions from baseline to endpoint in LS mean WFIRS-P total score were greater than one-half the SD at baseline in the Full Analysis Set (0.247; Table S1). Within each individual domain, the reductions in LS mean score were greater than one-half the SD of the domain score at baseline (Table S1) in the domains of Family, Learning and School, and Life Skills in the LDX group and in the domains of Learning and School and Life Skills in the ATX group.

\section{Discussion}

In this 9-week study, both LDX and ATX were associated with improvements in WFIRS-P total score and in scores across all six WFIRS-P domains in children and adolescents with ADHD who had experienced a clinically inadequate response to MPH therapy. The difference in these improvements between the LDX and ATX groups was statistically significant in favour of LDX for WFIRS-P total score and the Learning and School and the Social Activities domains, with effect sizes (LDX versus ATX) of 0.27, 0.43,

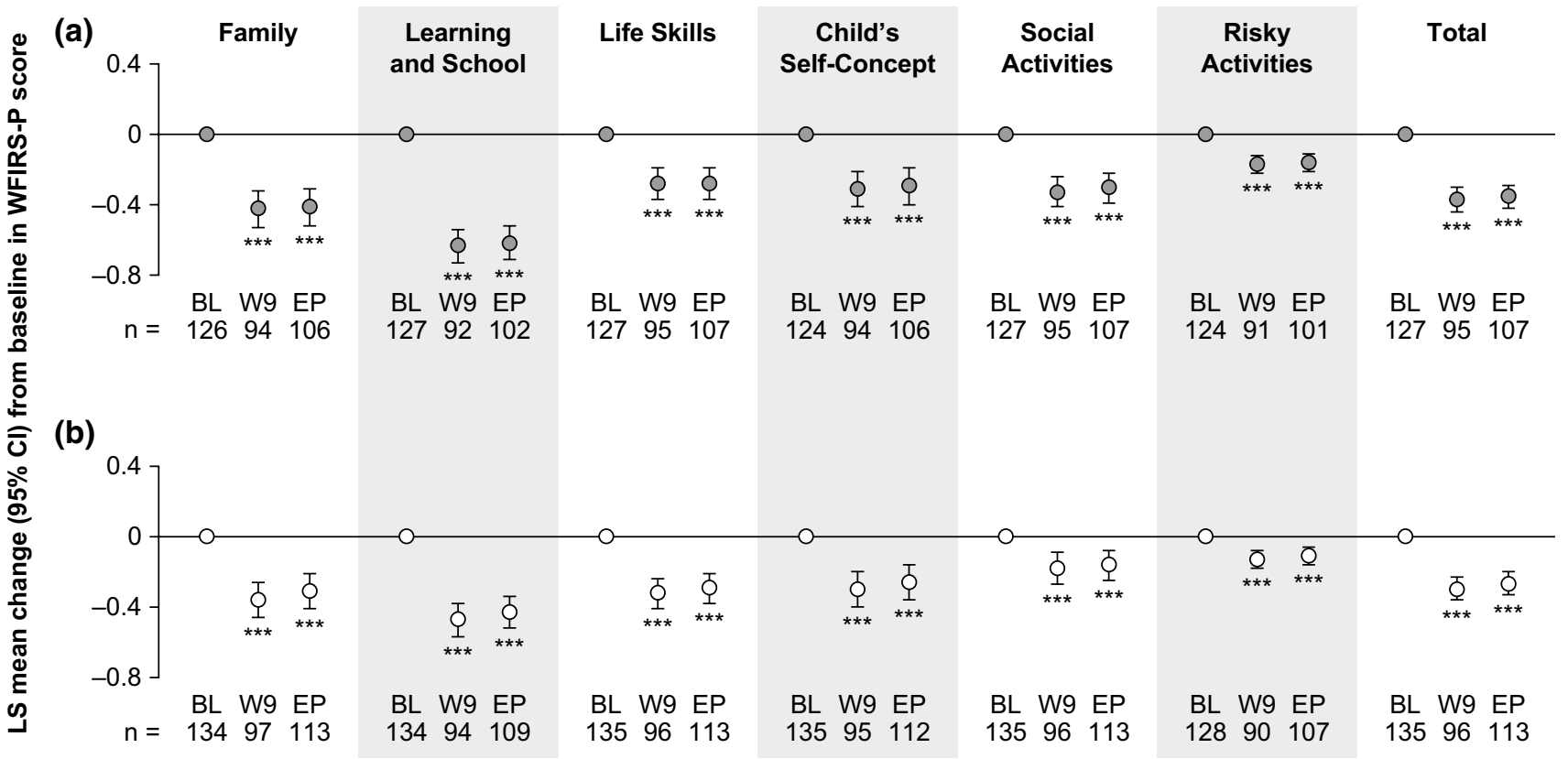

Fig. 2 Change in WFIRS-P scores from baseline to week 9 and endpoint in (a) the LDX treatment group and (b) the ATX treatment group. The number of observations $(n)$ shows the number of patients with a valid change from BL score at each time point. Endpoint was defined as the last on-treatment, post-baseline visit with a valid assessment. $p$ values are nominal and were not adjusted for multiple comparisons. $* * * p<0.001$ versus baseline (ad hoc analysis). ATX atomoxetine, $B L$ baseline, $C I$ confidence interval, $E P$ endpoint, $L D X$ lisdexamfetamine dimesylate, $L S$ least-squares, $n$ number of observations, W9 week 9, WFIRS-P Weiss Functional Impairment Rating Scale-Parent Report 
and 0.34 , respectively. These effect sizes are modest compared with the large effect size (0.924) of LDX versus placebo for improvement in WFIRS-P total score in a 7-week, randomized, double-blind, placebo-controlled study [5]. In the present head-to-head study, however, effect sizes are of LDX versus the active comparator, ATX, rather than versus placebo. Within each treatment group, the changes from baseline in WFIRS-P total score and some domain scores were greater than one-half SD for the population at baseline, suggesting that the observed improvements were clinically meaningful. However, the clinical relevance of the differences between active treatments in improvements in WFIRS-P scores remains to be established.

Depending on their age, patients with ADHD are more likely than their peers to underperform at school, to be unemployed or on a low income, to have accidents, to be arrested, to smoke or abuse substances, to become pregnant as teenagers, to contract sexually transmitted diseases, or to be divorced [1,23]. Indeed, functional impairment is a diagnostic criterion for ADHD [2, 3, 43], and its impact on patients' lives is commonly a major motivation for seeking treatment [37]. Therefore, effective treatments for ADHD should both improve symptoms and reduce the functional impairments that are associated with the disorder.

Relative to generic measures of function, disease-specific measures such as the WFIRS-P are believed to maximize sensitivity by focusing on particular areas of concern for patients with a given disorder and, as a result, may be especially useful for identifying and measuring treatment effects [21]. The sensitivity of the WFIRS-P to treatmentinduced changes in functioning has been demonstrated in a limited number of published clinical trials $[5,32,36$, 39]; however, population-based normative data are not yet available [29]. Nevertheless, several unpublished industrysponsored, government-funded or academically led interventional studies registered at clinicaltrials.gov specify the WFIRS-P as a secondary outcome measure, indicating its increasing acceptance as a measure of functional impairment outcomes in patients with ADHD [9-19].

Mean WFIRS-P total scores at baseline in the present study [LDX 0.95 (SD 0.474), ATX 0.91 (0.513)] were similar to those reported in two other published largescale trials. In a European, 7-week, double-blind, randomized, placebo- and active-controlled study of LDX in 336 children and adolescents with ADHD, mean WFIRS$\mathrm{P}$ total scores at baseline were in the range 1.01-1.10 (SD $0.437-0.456$ ) across the three treatment groups [5]. In an international, 12-month, open-label, randomized study of ATX compared with 'other standard therapy' in 398 children and adolescents with ADHD, mean WFIRS-P scores at baseline were 1.02 (SD 0.475) and $0.96(0.453)$ in the treatment groups, respectively [32]. All these baseline values exceed the optimal cut-off score $(0.65)$ for differentiating children with and without ADHD, according to receiver operating characteristic curve analysis of a multicentre observational study [5, 29]. Furthermore, in all three studies, the highest mean WFIRS-P scores at baseline were observed in the Family domain and the Learning and School domain (also referred to as the Home domain and School domain [32]), indicating that profound impairments in these areas of day-to-day functioning are a consistent finding in clinical studies of children and adolescents with ADHD.

Despite differences in study design and patient inclusion/exclusion criteria, the levels of improvement in WFIRS-P total and domain scores in the LDX group of the present 9-week head-to-head study were similar to those observed in the previous 7-week placebocontrolled study of LDX [5]. In both studies, the largest improvements from baseline in the LDX group were observed in the Learning and School domain, followed by the Family domain, and the smallest improvements were seen in the Life Skills and the Risky Activities domains. In the placebo-controlled study, the improvements from baseline were statistically significantly superior to placebo for WFIRS-P total score and the domains of Learning and School, Family, Social Activities, and Risky Activities. Some patients from the European placebo-controlled study, together with additional US patients, took part in a follow-up study of the longterm maintenance of efficacy of LDX [24], which also included the WFIRS-P as a secondary efficacy measure. In the open-label period of at least 26-week LDX treatment, WFIRS-P scores improved in all domains and in total [4]. In the subsequent 6-week, double-blind, placebo-controlled, randomized-withdrawal period, WFIRS-P scores deteriorated in the placebo group in all domains and in total, and the difference between the LDX and placebo groups was statistically significant for WFIRS-P total score and the domains of Family, Learning and School, and Risky Activities [4].

In the present study, the largest changes from baseline in the ATX group were observed in the Family domain and the Learning and School domain, as was also the case in the LDX group. These domains also saw the largest changes from baseline in both treatment arms of the 12-month open-label study comparing ATX with 'other standard therapy' (mainly MPH) [32] and in the osmotic-release oral system MPH (OROS-MPH) reference arm of the 7-week European, placebo-controlled study of LDX [5]. This reference arm was included to validate the study design and contextualize the results; statistical comparisons of OROSMPH versus LDX were not pre-specified [22].

Taken together, the available data therefore suggest that the Learning and School and the Family domains of the WFIRS-P are most responsive to therapy, as well as most 
affected at baseline. This does not necessarily mean, however, that the effects of different classes of ADHD medication on different domains of functional impairment are equivalent. For example, in the present study, LDX was statistically significantly superior to ATX in total score and in the Social Activities and the Learning and School domains, but not in other domains. Also, in the 7-week, European, placebocontrolled study of LDX, the effect sizes for WFIRS-P total score of LDX versus placebo and of the OROS-MPH reference treatment versus placebo were both large $(0.924$ and 0.772 , respectively), but the placebo-adjusted effects of the two medications were not identical across the six WFIRS-P domains [5] (no post hoc statistical analysis comparing LDX versus OROS-MPH has been published for these outcomes, in contrast to the primary efficacy outcome [38]). The potential implications of these findings and the present results for individualized patient management remain to be established.

Although the present study did not investigate the time course of changes in WFIRS-P scores, evidence from previous studies suggests that the rate of response to treatment may differ across WFIRS-P domains. First, within both the LDX arm and OROS-MPH reference arm of the European, placebo-controlled study of LDX, some domains did not show improvement from baseline until week 7 , whereas improvement from baseline in others was evident at week 4 [5]. Secondly, most, but not all, of the improvement from baseline in WFIRS-P scores in the open-label period of the subsequent randomized-withdrawal study occurred before week 8 [4]. Thirdly, in the long-term, open-label comparison of ATX with 'other standard therapy', the only statistically significant difference in WFIRS-P scores between groups was in favour of 'other standard therapy' in the Learning and School domain at 6 months $(p<0.05)$; this difference was not evident after 12 months of treatment [32]. These observations suggest that some domains of functional impairment may be more likely than other aspects of ADHD to respond to pharmacotherapy over a longer treatment period than was used in the present study.

The associations between ADHD symptoms, functional impairments, and health-related quality of life (HRQoL) have yet to be fully understood. HRQoL instruments measure a patient's (or a parent's) subjective perception of the impact of health status on their (or their child's) physical, psychological, and social well-being [26]. Studies investigating the correlations between ADHD symptom severity ratings and HRQoL scores have revealed statistically significant, but only moderately strong, correlations between the two measures $(r=0.2-0.6)$, supporting the view that symptoms and HRQoL represent related but distinct constructs [26]. There are, however, few data available to establish the interactions between functional impairment and either ADHD symptoms or HRQoL. Analyses have indicated a statistically significant, but imperfect, correlation between functioning and
HRQoL, suggesting that these too are related but distinct constructs [20]. This is consistent with the observation in the present study that the effect sizes of LDX versus ATX for WFIRS-P scores (total score 0.27, Learning and School 0.43 , Social Activities 0.34, all $p<0.05$ ) were smaller than for the previously reported ADHD-RS-IV symptomatological outcome measure $(0.56 ; p<0.001)$ [27]. Effect sizes for LDX and OROS-MPH versus placebo were also larger for ADHD-RS-IV total score than for WFIRS-P scores in the previous 7-week, European, placebo-controlled study [5, 22]. Further studies and analyses are required to increase the understanding of the interrelationships between ADHD symptoms, functional impairment, and HRQoL.

The safety outcomes of the present study have been published previously and were consistent with those of earlier clinical trials [27]. In summary, treatment-emergent adverse events were reported by similar proportions of patients in each treatment group (LDX $71.9 \%$, ATX $70.9 \%$ ). [27] Weight loss was larger in the LDX group than in the ATX group, and the proportion of patients who met the outlier criterion for high pulse rate ( $\geq 100$ beats per minute) was higher in the ATX group than in the LDX group [27]. With these exceptions, the changes in mean vital signs and electrocardiogram parameters, the proportions of patients meeting other outlier criteria, and the frequency of potentially clinically important vital sign observations were all similar for LDX and ATX treatment [27].

Strengths of the present study include the sample size, the head-to-head, randomized, double-blind, doseoptimized design, and the geographically diverse patient population. Patients were required to have experienced an inadequate response to MPH therapy, making the present findings particularly relevant in European countries in which LDX is approved for the treatment of children and adolescents when response to previous MPH treatment is considered clinically inadequate by the supervising specialist. Although the definition of inadequate response to MPH therapy was broad, lack of efficacy was the most common reason given for an inadequate response.

An important limitation of the study is the relatively short duration of treatment, which precludes evaluation of the long-term effects of treatment on functional impairment. The observation that it may take 12 or more weeks for ATX to reach its maximum effect on ADHD symptoms [42] suggests the possibility that the 9-week duration of the study may have led to an underestimation of the efficacy of ATX. Improvements in functioning may occur over a longer treatment period than relief of symptoms, potentially also leading to underestimation of the effects of treatment on functional impairment as a secondary outcome in the present study. Furthermore, although the dose-titration schedules of both treatments followed current guidelines, twicedaily ATX and/or higher doses of ATX may have been more 
effective than the once-daily dosing required in the present study [35]. Finally, the possibility cannot be excluded that patients' experience of MPH treatment before enrolment may have led to preconceptions regarding the ADHD medications under investigation (perhaps relating to their onset or duration of efficacy or tolerability), which might have influenced functional outcomes in this double-blind study.

In conclusion, this study has shown that both LDX and ATX treatment can improve functioning, as measured by the WFIRS-P, in children and adolescents with ADHD who have experienced a clinically inadequate response to MPH (as judged by investigators). Improvements overall and in certain domains were statistically significantly greater in magnitude with LDX treatment than with ATX treatment, within the time frame of the study. These findings may aid clinicians when developing treatment plans to address the functional impairments associated with ADHD.

Acknowledgments Study SPD489-317 was funded by Shire Development LLC. Shire develops and markets drugs to treat psychiatric disorders, including ADHD. The authors thank the patients and their parents/guardians and the investigators who took part in the study, including the coordinating principal investigator, Professor Ralf W Dittmann (Paediatric Psychopharmacology, Department of Child and Adolescent Psychiatry and Psychotherapy, Central Institute of Mental Health, Medical Faculty Mannheim, University of Heidelberg, Mannheim, Germany). E Cardo, DR Coghill, A Häge, and P Nagy were investigators or principal investigators in this clinical study. CS Anderson, B Caballero, and V Sikirica contributed to the study design. B Adeyi was responsible for the statistical analysis. All authors were involved in discussion and interpretation of the data, critically revised the article, and approved the manuscript for submission. Shire International $\mathrm{GmbH}$ provided funding to Oxford PharmaGenesis ${ }^{\mathrm{TM}} \mathrm{Ltd}$ for writing and editorial support for this publication. Dr T Gristwood and Dr E Southam of Oxford PharmaGenesis ${ }^{\mathrm{TM}}$ Ltd provided writing assistance under the direction of the authors. Editorial assistance in editing, fact checking, formatting, proofreading, and submission was also provided by Oxford PharmaGenesis ${ }^{\mathrm{TM}} \mathrm{Ltd}$.

Conflict of interest B Adeyi, CS Anderson, B Caballero, and V Sikirica are employees of Shire and own stock/stock options. The following authors have received compensation for serving as consultants or speakers, or they or the institutions they work for have received research support or royalties, from the companies or organizations indicated: A Häge [Janssen-Cilag, Lilly, Otsuka, Shire, German Research Foundation (DFG), German Ministry of Education and Research (BMBF), European Union (EU FP7 programme)]; E Cardo (Eli Lilly, Health Spanish Ministry Research Fund, Ministry of Education Grant Research, Shire, UCB); DR Coghill (Flynn Pharma, Janssen-Cilag, Lilly, Medice, Novartis, Otsuka, Oxford University Press, Pfizer, Schering-Plough, Shire, UCB, Vifor Pharma); P Nagy (Tourette Syndrome Association of the USA, Hungarian Ministry of Education, National Development Agency of Hungary, Otsuka, Shire).

Open Access This article is distributed under the terms of the Creative Commons Attribution 4.0 International License (http://creativecommons.org/licenses/by/4.0/), which permits unrestricted use, distribution, and reproduction in any medium, provided you give appropriate credit to the original author(s) and the source, provide a link to the Creative Commons license, and indicate if changes were made.

\section{References}

1. Adler AL (2011) Optimizing clinical outcomes across domains of life in adolescents and adults with ADHD. J Clin Psychiatry 72:1008-1014

2. American Psychiatric Association (2013) Diagnostic and statistical manual of mental disorders, fifth edition (DSM-5). American Psychiatric Publishing, Arlington

3. American Psychiatric Association (2000) Diagnostic and statistical manual of mental disorders, fourth edition, text revision (DSM-IV-TR). The Press, Washington, DC

4. Banaschewski T, Johnson M, Lecendreux M, Zuddas A, Adeyi B, Hodgkins P, Squires L, Coghill D (2014) Health-related quality of life and functional outcomes from a randomized-withdrawal study of long-term lisdexamfetamine dimesylate treatment in children and adolescents with attention-deficit/hyperactivity disorder. CNS Drugs 28:1191-1203

5. Banaschewski T, Soutullo C, Lecendreux M, Johnson M, Zuddas A, Hodgkins P, Adeyi B, Squires LA, Coghill D (2013) Healthrelated quality of life and functional outcomes from a randomized, controlled study of lisdexamfetamine dimesylate in children and adolescents with attention deficit hyperactivity disorder. CNS Drugs 27:829-840

6. Barkley RA (2002) Major life activity and health outcomes associated with attention-deficit/hyperactivity disorder. J Clin Psychiatry 63(Suppl 12):10-15

7. Biederman J, Boellner SW, Childress A, Lopez FA, Krishnan S, Zhang Y (2007) Lisdexamfetamine dimesylate and mixed amphetamine salts extended-release in children with ADHD: a double-blind, placebo-controlled, crossover analog classroom study. Biol Psychiatry 62:970-976

8. Biederman J, Krishnan S, Zhang Y, McGough JJ, Findling RL (2007) Efficacy and tolerability of lisdexamfetamine dimesylate (NRP-104) in children with attention-deficit/hyperactivity disorder: a phase III, multicenter, randomized, double-blind, forceddose, parallel-group study. Clin Ther 29:450-463

9. ClinicalTrials.gov (2014) Dose-optimization in adolescents aged 13-17 diagnosed with attention-deficit/hyperactivity disorder (ADHD) using extended-release guanfacine HCl. http://ClinicalTrials.gov/show/NCT01081132. Accessed 2 July 2014

10. ClinicalTrials.gov (2013) Efficacy and safety of extended-release guanfacine hydrochloride in children and adolescents aged 6-17 years with attention-deficit/hyperactivity disorder (ADHD). http://ClinicalTrials.gov/show/NCT01244490. Accessed 2 July 2014

11. ClinicalTrials.gov (2013) Efficacy and safety of Kapvay ${ }^{\mathrm{TM}}$ extended-release in children and adolescents with attentiondeficit/hyperactivity disorder. http://ClinicalTrials.gov/show/ NCT01439126. Accessed 2 July 2014

12. ClinicalTrials.gov (2014) Efficacy study of polyunsaturated fatty acids in children and adolescents with attention deficit/hyperactivity disorder. http://ClinicalTrials.gov/show/NCT01340690. Accessed 2 July 2014

13. ClinicalTrials.gov (2014) Enhancement of methylphenidate treatment by psychosocial intervention and support. http://ClinicalTrials.gov/show/NCT01660425. Accessed 2 July 2014

14. ClinicalTrials.gov (2014) An exploratory, single-blind pilot study of flexible doses of the triple reuptake inhibitor EB-1020 $\mathrm{SR}$ in the treatment of adult males with attention-deficit/hyperactivity disorder. http://ClinicalTrials.gov/show/NCT01939353. Accessed 2 July 2014

15. ClinicalTrials.gov (2014) Follow-up treatment of children with attention-deficit/hyperactivity disorder (ADHD). http://ClinicalTrials.gov/show/NCT02142140. Accessed 2 July 2014

16. ClinicalTrials.gov (2013) Lisdexamfetamine dimesylate in the treatment of adult ADHD with anxiety disorder comorbidity. 
http://ClinicalTrials.gov/show/NCT01863459. Accessed 2 July 2014

17. ClinicalTrials.gov (2014) Maintenance of efficacy of extendedrelease guanfacine $\mathrm{HCl}$ in children and adolescents with attention-deficit/hyperactivity disorder (ADHD). http://ClinicalTrials. gov/show/NCT01081145. Accessed 2 July 2014

18. ClinicalTrials.gov (2009) Stimulant versus non-stimulant medication for attention-deficit/hyperactivity disorder in children. http://ClinicalTrials.gov/show/NCT00183391. Accessed 2 July 2014

19. ClinicalTrials.gov (2014) Tolerability and efficacy of AM and PM once-daily dosing with extended-release guanfacine hydrochloride in children 6-12 with attention-deficit/hyperactivity disorder (ADHD) (the ADHD Tempo Study). http://ClinicalTrials. gov/show/NCT00997984. Accessed 2 July 2014

20. Coghill D (2010) The impact of medications on quality of life in attention-deficit hyperactivity disorder: a systematic review. CNS Drugs 24:843-866

21. Coghill D (2011) Pragmatic measures in paediatric psychopharmacology-are we getting it right? Eur Neuropsychopharmacol 21:571-583

22. Coghill D, Banaschewski T, Lecendreux M, Soutullo C, Johnson M, Zuddas A, Anderson C, Civil R, Higgins N, Lyne A, Squires L (2013) European, randomized, phase 3 study of lisdexamfetamine dimesylate in children and adolescents with attentiondeficit/hyperactivity disorder. Eur Neuropsychopharmacol 23:1208-1218

23. Coghill D, Soutullo C, d'Aubuisson C, Preuss U, Lindback T, Silverberg M, Buitelaar J (2008) Impact of attention-deficit/ hyperactivity disorder on the patient and family: results from a European survey. Child Adolesc Psychiatry Ment Health 2:31

24. Coghill DR, Banaschewski T, Lecendreux M, Johnson M, Zuddas A, Anderson CS, Civil R, Dauphin M, Higgins N, Lyne A, Gasior M, Squires LA (2014) Maintenance of efficacy of lisdexamfetamine dimesylate in children and adolescents with attention-deficit/hyperactivity disorder: randomized-withdrawal study design. J Am Acad Child Adolesc Psychiatry 53(647-657):e641

25. Cohen J (1992) A power primer. Psychol Bull 112:155-159

26. Danckaerts M, Sonuga-Barke EJ, Banaschewski T, Buitelaar J, Dopfner M, Hollis C, Santosh P, Rothenberger A, Sergeant J, Steinhausen HC, Taylor E, Zuddas A, Coghill D (2010) The quality of life of children with attention deficit/hyperactivity disorder: a systematic review. Eur Child Adolesc Psychiatry 19:83-105

27. Dittmann RW, Cardo E, Nagy P, Anderson CS, Bloomfield R, Caballero B, Higgins N, Hodgkins P, Lyne A, Civil R, Coghill D (2013) Efficacy and safety of lisdexamfetamine dimesylate and atomoxetine in the treatment of attention-deficit/hyperactivity disorder: a head-to-head, randomized, double-blind, phase IIIb study. CNS Drugs 27:1081-1092

28. DuPaul GJ, Power T, Anastopoulos A, Reid R (1998) The ADHD rating scale-IV, checklist, norms, and clinical interpretation. Guildford Press, New York

29. Epstein JN, Weiss MD (2012) Assessing treatment outcomes in attention-deficit/hyperactivity disorder: a narrative review. Prim Care Companion CNS Disord 14:PCC.11r01336

30. European Medicines Agency, Committee for Medicinal Products for Human Use (2010) Guideline on the clinical investigation of medicinal products for the treatment of attention deficit hyperactivity disorder (ADHD). http://www.ema.europa.eu/docs/en_GB/
document_library/Scientific_guideline/2010/08/WC500095686. pdf. Accessed 22 November 2013

31. Findling RL, Childress AC, Cutler AJ, Gasior M, Hamdani M, Ferreira-Cornwell MC, Squires L (2011) Efficacy and safety of lisdexamfetamine dimesylate in adolescents with attention-deficit/hyperactivity disorder. J Am Acad Child Adolesc Psychiatry 50:395-405

32. Fuentes J, Danckaerts M, Cardo E, Puvanendran K, Berquin P, De Bruyckere K, Montoya A, Quail D, Escobar R (2013) Longterm quality-of-life and functioning comparison of atomoxetine versus other standard treatment in pediatric attention-deficit/ hyperactivity disorder. J Clin Psychopharmacol 33:766-774

33. Guy W (ed) (1976) Clinical global impressions (CGI). In: ECDEU assessment manual for psychopharmacology. US Department of Health, Education, and Welfare, Rockville, MD

34. Guyatt GH, Osoba D, Wu AW, Wyrwich KW, Norman GR, Clinical Significance Consensus Meeting G (2002) Methods to explain the clinical significance of health status measures. Mayo Clin Proc 77:371-383

35. Hanwella R, Senanayake M, de Silva V (2011) Comparative efficacy and acceptability of methylphenidate and atomoxetine in treatment of attention deficit hyperactivity disorder in children and adolescents: a meta-analysis. BMC Psychiatry 11:176

36. Maziade M, Rouleau N, Lee B, Rogers A, Davis L, Dickson R (2009) Atomoxetine and neuropsychological function in children with attention-deficit/hyperactivity disorder: results of a pilot study. J Child Adolesc Psychopharmacol 19:709-718

37. Parens E, Johnston J (2009) Facts, values, and attention-deficit hyperactivity disorder (ADHD): an update on the controversies. Child Adolesc Psychiatry Ment Health 3:1

38. Soutullo C, Banaschewski T, Lecendreux M, Johnson M, Zuddas A, Anderson C, Civil R, Higgins N, Bloomfield R, Squires LA, Coghill DR (2013) A post hoc comparison of the effects of lisdexamfetamine dimesylate and osmotic-release oral system methylphenidate on symptoms of attention-deficit hyperactivity disorder in children and adolescents. CNS Drugs 27:743-751

39. Stein MA, Waldman ID, Charney E, Aryal S, Sable C, Gruber R, Newcorn JH (2011) Dose effects and comparative effectiveness of extended release dexmethylphenidate and mixed amphetamine salts. J Child Adolesc Psychopharmacol 21:581-588

40. The Canadian Attention Deficit Hyperactivity Disorder Resource Alliance (CADDRA) (2011) Weiss Functional Impairment Rating Scale - Parent Report (2011 version). http://www.caddra. ca/cms4/pdfs/caddraGuidelines2011WFIRS_P.pdf. Accessed 13 June 2014

41. Weiss MD, Wasdell MB, Bomben MM (2004) Weiss Functional Impairment Rating Scale - Parent Report (Version 2). http://www.adhd-monitoring.com/WFIRSParentReport-3.pdf. Accessed 13 June 2011

42. Wilens TE, Newcorn JH, Kratochvil CJ, Gao H, Thomason CK, Rogers AK, Feldman PD, Levine LR (2006) Long-term atomoxetine treatment in adolescents with attention-deficit/hyperactivity disorder. J Pediatr 149:112-119

43. World Health Organization (2010) International statistical classification of diseases and related health problems, ICD-10. World Health Organization, Geneva

44. Wyrwich KW, Tierney WM, Wolinsky FD (1999) Further evidence supporting an SEM-based criterion for identifying meaningful intra-individual changes in health-related quality of life. J Clin Epidemiol 52:861-873 